because other tests like microscopy or molecular analysis are not commonly available. Commonly used and sensitive malaria RDTs exploit a specific antigen from P. falciparum, the histidine-rich protein-2 (HRP2). Studies performed in different parts of the world showed HRP2-deficient P. falciparum strains which cause malaria infections that are not detected by the commonly used RDTs. This factor is threatening WHO efforts to control malaria worldwide. Studies performed in neighboring Eritrea and Kenya showed high HRP2 deficiency rates in the studied populations. To date, no data are available from Ethiopia.

Starting during the summer of 2018, blood samples from microscopy-positive Falciparum malaria patients living in two highly endemic areas for malaria in the Rift Valley (Ziway Dugda and Abomsaa) are being investigated by RDT and subsequent molecular analyses for HRP2 (and HRP3) genes are being done. To perform the needed investigations, PCR techniques are being established and training on the methodology is given at the Hirsch Institute for cooperating scientists and technicians.

\section{Cooperation Projects with Other Institutes and Universities}

The HITM endeavors to promote scientific exchange on a national as well as an international level. For this purpose, the employees of the institute support the activities of other research facilities by helping them organize studies and acquire sample materials. The coordinators of the institute are in contact with several na- tional and international medical research establishments.

These include the Friedrich Löfler Institute in Greifswald, the Armauer Hansen Research Institute in Addis Ababa, the Ethiopian Health and Nutrition Research Institute, the Ethiopian Biodiversity Institute, the Oromia Regional Laboratory in Adama, Black Lion Hospital in Addis Ababa, Adama Hospital, Bekoji Regional Hospital, Adama Science and Technology University, and Jimma University. Within the ESTHER project "Prevention and clinical management of hospital-acquired infections at Asella Teaching Hospital, Central Ethiopia", joint workshops, training and research activities are conducted with other partners of the ESTHER network (e.g. German institutions from Hamburg, Munich, Frankfurt, and Berlin and their African partners from Rwanda, Tanzania, Ethiopia, Kenya, Ivory Coast, and Ghana). Within this network, further training of physicians and academic employees of the ATH is frequently possible. For example, in May 2018 one physician, one microbiologist, and one pharmacist from the ATH participated in the workshop on antimicrobial stewardship held by ESTHER partners at Kenyatta Hospital in Nairobi, Kenya.

\section{Recognition from the Ethiopian Ministry of Science and Technology}

At the Ethiopian Ministry of Science and Technology (MoST) and the affiliated National Ethics Review Board (NERB) it was noticed that many applications for approval of clinical studies in Ethiopia were submitted from a previously unknown study site - the Hirsch 